\title{
COMPLICAÇÕES RESPIRATÓRIAS PÓS-OPERATÓRIAS: UMA ANÁLISE SOCIODEMOGRÁFICA DOS PACIENTES INTERNADOS EM UM HOSPITAL DO ALTO SERTÃO PARAIBANO
}

\author{
POSTOPERATIVE RESPIRATORY COMPLICATIONS: A SOCIO- \\ DEMOGRAPHICAL ANALYSIS OF PATIENTS HOSPITALIZED IN A \\ HOSPITAL OF ALTO SERTÃO PARAIBANO
}

\author{
Paulo André Guerra Calazans ${ }^{1}$ \\ José Liberlando Alves de Albuquerque ${ }^{2}$ \\ Renata Lívia Silva Fonseca Moreira de Medeiros ${ }^{3}$ \\ Fabrício dos Santos Menezes ${ }^{4}$ \\ Fernanda de Souza Maurício ${ }^{5}$
}

RESUMO: INTRODUÇÃO: A utilização dos serviços de saúde é uma expressão positiva do acesso, que pode ser medida através da assistência de rotina ou emergência. Diante disso, o adequado planejamento de ações cirúrgicas vincula-se à ausência de complicações perioperatórias. A ocorrência de complicações respiratórias no pós-operatório representa um risco substancial à morbidade, internações hospitalares longas e aumento dos gastos em saúde pública. Sendo assim, a geração de informações de base populacional possibilita monitorar o acesso aos serviços, avaliar e aperfeiçoar a assistência à saúde. O conhecimento sociodemográfico dos usuários e a ocorrência das complicações respiratórias pósoperatórias podem permitir uma intervenção mais eficaz, com redução das taxas de morbimortalidade, redução do tempo de internamento hospitalar e redução dos gastos em saúde pública. OBJETIVO: Caracterizar os pacientes submetidos a procedimentos cirúrgicos, identificar o perfil epidemiológico e apontar as complicações respiratórias pós-operatórias. METODOLOGIA: Trata-se de um estudo transversal, descritivo, de abordagem quantitativa, realizado com dados dos prontuários de pacientes submetidos a procedimentos cirúrgicos no Hospital Regional de Cajazeiras/PB em 2017. RESULTADOS: O perfil sociodemográfico mostrou a maioria dos pacientes do sexo masculino e com faixa etária predominante

\footnotetext{
1 Discente de Bacharelado em Medicina da Faculdade Santa Maria.

2 Docente de Bacharelado em Medicina da Faculdade Santa Maria.

3 Docente de Bacharelado em Medicina da Faculdade Santa Maria.

4 Doutorando em Epidemiologia da Universidade de São Paulo.

${ }^{5}$ Discente de Bacharelado em Medicina da Faculdade Santa Maria.
} 
de adultos jovens, com idade até 39 anos, sendo a frequência de tabagistas ativos consonante com a média da população brasileira. Apenas $10 \%$ da amostra evoluiu com alguma complicação respiratória no pós-operatório. CONCLUSÕES: A partir dos resultados obtidos, é possível inferir que as características encontradas podem contribuir para a organização da assistência, uma vez que permitem identificar os grupos populacionais mais vulneráveis. No entanto, o presente estudo é limitado por incluir poucas variáveis sociodemográficas, sendo necessários estudos mais complexos e com mais variáveis a serem analisadas, para ampliar a compreensão da realidade e perfil dos usuários do SUS em nível regional.

Descritores: Complicações. Fatores de risco. Perfil epidemiológico.

ABSTRACT: INTRODUCTION: The use of health service is a positive expression of access, which can be measured through routine or emergency care. Therefore, the appropriate planning of surgical actions is linked to the absence of perioperative complications. Postoperative respiratory complications represent a substantial risk to morbidity, long hospital stays and increased public health spending. Thus, the generation of population-based information allows for monitoring access to services, evaluating and improving health care. Sociodemographic knowledge of users and the occurrence of postoperative respiratory complications may allow for a more effective intervention, reducing morbidity and mortality rates, reducing hospital stay and reducing public health expenses. OBJECTIVE: To characterize the patients submitted to surgical procedures, to identify the epidemiological profile and to point out the postoperative respiratory complications. METHODOLOGY: This is a crosssectional, descriptive and quantitative study, conducted with data from medical records of patients submitted to surgical procedures at Cajazeiras Regional Hospital/PB in 2017. RESULTS: The sociodemographic profile showed the majority of male patients with a predominant age group of young adults, aged up to 39 years, and the frequency of active smokers is in line with the average of the Brazilian population. Only $10 \%$ of the sample evolved with any postoperative respiratory complications. CONCLUSIONS: The obtained results showed that the characteristics found can contribute to the organization of care, since they allow for the identification of the most vulnerable population groups. However, the present study is limited because it includes few sociodemographic variables, requiring more complex studies with more variables for analysis to broaden the understanding of the reality and profile of the UHS users at the regional level.

Descriptors: Complications. Risk factors. Epidemiological profile. 


\section{INTRODUÇÃO}

O acesso ao sistema de saúde pode ser medido pela utilização de serviços de rotina ou de emergência. Dessa forma, a utilização de serviços de saúde é uma expressão positiva do acesso, ao impactar na saúde das populações, prevenindo a ocorrência de doenças, reduzindo a mortalidade e aumentando a sobrevivência (SILVA et al., 2011). A partir desse raciocínio, subsídios que possibilitem o planejamento das ações cirúrgicas são essenciais e vinculam-se à ausência de complicações no pós-operatório.

A elevada ocorrência de complicações respiratórias em pacientes submetidos a procedimentos cirúrgicos é relevante por apresentar um risco à morbidade, internações hospitalares mais longas e elevação dos gastos em saúde pública (LANGERON et al., 2014). Pode apresentar índices de mortalidade de até $25 \%$, a depender do procedimento cirúrgico e tipo de complicação (TAYLOR; DEBOARD; GAUVIN, 2015).

Mesmo com avanços nos cuidados perioperatórios, a prevalência de complicações respiratórias no pós-operatório pode variar de 2 a 40\%, segundo o trabalho realizado por Silva, Gazzana e Knorst (2010), sendo essa ampla variação atribuída às divergências na literatura no que se refere às alterações fisiopatológicas esperadas daquilo que pode ser considerado complicação pulmonar. De acordo com Avila e Fenili, (2017), são o segundo tipo mais comum, após as complicações relacionadas ao sítio cirúrgico, podendo ser tão prevalentes quanto às cardíacas. Nesse aspecto, traçar o perfil sociodemográfico é importante para a devida estratificação do risco, elaboração de medidas preventivas e cuidados perioperatórios mais específicos, quando necessários (YANG et al., 2015).

Deve-se destacar que, antes mesmo de ser submetido a um procedimento cirúrgico, o paciente realiza uma série de exames pré-operatórios, a fim de reduzir as chances de alguma intercorrência. Todavia, como exposto, estes exames não anulam a presença de alguma complicação; eles apenas mostram em que situação 
encontra-se a saúde do paciente e se ele/ela está apto(a) à cirurgia. Como seres vivos cujos metabolismo e fisiologia alteram-se de tempos em tempos, no momento da técnica cirúrgica, poderá haver alguma intercorrência.

Além disso, existem doenças que se encontram na forma subclínica, estando, de certa forma, mascaradas, ou seja, o paciente possui alguma enfermidade, porém a mesma não apresenta quaisquer sintomas ou alterações. Estas doenças também podem contribuir para uma complicação peri e pós-operatória. Existem fatores de risco que contribuem para a ocorrência de complicações respiratórias pósoperatórias. Neste grupo, inserem-se idade avançada, saturação de oxigênio préoperatória reduzida, ocorrência de infecção respiratória no mês anterior, anemia préoperatória, incisão cirúrgica próxima ao diafragma, maior tempo de cirurgia e cirurgias de emergência. Outros fatores incluem estado funcional reduzido, estado mental alterado, perda de peso maior que $10 \%$ nos últimos seis meses, doença renal crônica, diabetes mellitus, insuficiência cardíaca congestiva e abuso de álcool (TAYLOR; DEBOARD; GAUVIN, 2015).

A anestesia geral também é responsável por contribuir para intercorrências respiratórias pós-operatórias, uma vez que é capaz de alterar a função mucociliar, promovendo a retenção de secreções, causa broncoconstrição, reduz a produção de substância surfactante e inibe a atividade dos macrófagos alveolares (TAYLOR; DEBOARD; GAUVIN, 2015). Neste contexto, uma avaliação pré-operatória completa e eficaz torna-se de suma importância para minimizar ao máximo os riscos de quaisquer tipos de complicações.

Ademais, existem populações que são consideradas mais susceptíveis às complicações respiratórias. Tais grupos incluem pessoas obesas, com apneia obstrutiva do sono (SAOS), síndrome de hipoventilação por obesidade (SHO), asma e doença pulmonar obstrutiva crônica (DPOC). Destaca-se, ainda, que a maioria das pessoas que apresenta SHO também possuem SAOS (TAYLOR; DEBOARD; GAUVIN, 2015).

Da mesma forma, condições e quadros pós-operatórios podem desencadear tais complicações, como a inibição dos músculos respiratórios devido à dor na incisão, interrupção funcional em decorrência da incisão e inibição do reflexo da 
produção dos neurônios motores frênicos da tração de órgãos abdominais (TAYLOR; DEBOARD; GAUVIN, 2015).

As principais intercorrências respiratórias pós-operatórias envolvem pneumonia, traqueobronquite, atelectasia com repercussão clínica, insuficiência respiratória aguda, intubação traqueal prolongada, ventilação mecânica prolongada, broncoespasmo, embolia pulmonar, edema pulmonar, pneumotórax ou derrame pleural, e óbito por complicação respiratória (SILVA; GAZZANA; KNORST, 2010).

É importante ressaltar que a geração de informações de base populacional, especificamente no âmbito regional, constitui um instrumento capaz de monitorar o acesso aos serviços, permitindo avaliar e fornecer subsídios para o aperfeiçoamento do serviço de saúde. No que concerne ao contexto das complicações pósoperatórias, existe a preocupação relacionada com as taxas de morbidade, mortalidade e à operacionalização dos gastos pelo Sistema único de Saúde (SUS), vislumbrando a possibilidade de intervenções mais eficazes. Sendo assim, o objetivo desse trabalho foi verificar o perfil sociodemográfico dos pacientes que utilizam o serviço de cirurgia geral do Hospital Regional de Cajazeiras/PB, mais especificamente, analisar os dados referentes ao grupo etário, gênero e tabagismo ativo crônico, relacionando-os à ocorrência de complicações respiratórias no pósoperatório.

\section{MATERIAIS E MÉTODOS}

O estudo foi realizado a partir da análise retrospectiva, transversal, descritiva, com abordagem quantitativa, de prontuários dos pacientes submetidos a procedimentos cirúrgicos do serviço de cirurgia geral do Hospital Regional de Cajazeiras(HRC)/PB, no período correspondente ao ano de 2017. A coleta dos dados foi realizada somente após anuência do Comitê de Ética em Pesquisa da Faculdade Santa Maria, conforme a resolução 466/12 do Conselho Nacional de Saúde. Foram 333 pacientes atendidos pelo serviço de cirurgia geral do HRC, com realização de procedimentos em nível de centro cirúrgico, que se enquadraram nos 
critérios de inclusão (idade superior a 18 anos, mínimo 48 horas de internação hospitalar, ausência de complicações respiratórias agudas no pré-operatório). Para isso, foi utilizado um instrumento próprio contendo questões referentes à caracterização sociodemográfica dos pacientes.

A análise retrospectiva consiste em analisar dados já coletados a fim de reunir informações a respeito de determinada população. Possui característica transversal por analisar dados de um período específico; possui natureza descritiva por ter o objetivo de descrever características de um fenômeno específico (neste caso, as complicações respiratórias pós-cirúrgicas) e com abordagem quantitativa porque se foca em descrever numericamente os dados.

Vale ressaltar que não houve necessidade de assinatura do Termo de Consentimento Livre e Esclarecido por se tratar de prontuários de pacientes já atendidos, não sendo expostos a riscos ou benefícios inerentes às pesquisas que lidam diretamente como ser humano. Entretanto, o sigilo das informações pessoais dos pacientes foi mantido, divulgando-se, apenas, os dados de interesse do estudo e que não comprometessem ou que pudessem trazer qualquer prejuízo aos indivíduos atendidos.

\section{RESULTADOS}

A partir da análise dos prontuários coletados $(n=333)$, foi possível realizar uma análise descritiva do perfil sociodemográfico da amostra (Tabela 1). Dos 333 pacientes incluídos no estudo, 57\% (190) eram do sexo masculino e $43 \%$ (143), do sexo feminino. Com idade variando entre 18 e 101 anos, obtendo-se maior frequência entre os adultos jovens até 39 anos, representando 39,6\% (132) da amostra; 30,6\% (102) da amostra são compostos por adultos com idade entre 40 e 59 anos, sendo seguidos por 29,7\% (99) de idosos, com idade igual ou superior a 60 anos. 


\begin{tabular}{|c|c|c|c|c|}
\hline \multirow[b]{2}{*}{ Variável } & \multirow[b]{2}{*}{ Categoria } & \multicolumn{3}{|c|}{ Complicação } \\
\hline & & $\frac{\text { Sim }}{N(\%)}$ & $\begin{array}{c}\text { Não } \\
\text { N (\%) }\end{array}$ & $\begin{array}{l}\text { Total } \\
\text { N (\%) }\end{array}$ \\
\hline \multicolumn{5}{|c|}{$\begin{array}{l}\text { Características dos } \\
\text { pacientes }\end{array}$} \\
\hline \multirow[t]{3}{*}{ Faixa etária } & $0-39$ anos & $7(5,3)$ & $125(94,7)$ & $132(100,0)$ \\
\hline & 40-59 anos & $7(6,9)$ & $95(93,1)$ & $102(100,0)$ \\
\hline & $\geq 60$ anos & $20(20,2)$ & $79(79,8)$ & $99(100,0)$ \\
\hline \multirow[t]{2}{*}{ Sexo } & Feminino & $9(6,3)$ & $134(93,7)$ & $143(100,0)$ \\
\hline & Masculino & $25(13,2)$ & $165(86,8)$ & $190(100,0)$ \\
\hline
\end{tabular}

Tabela 1. Estruturas etária e de gênero da amostra.

A partir da amostra analisada, $34(10,2 \%)$ pacientes evoluíram com, ao menos uma complicação respiratória pós-operatória, sendo $9(6,3 \%)$ do sexo feminino e 25 $(13,2 \%)$ do sexo masculino. Desses casos acometidos, 19 (55,9\%) evoluíram com mais de uma complicação.

No que diz respeito ao tabagismo ativo (Tabela 2), foi verificado que 15,6\% (52) da amostra autodeclararam serem tabagistas ativos e $84,4 \%$ (281) dos pacientes afirmaram que nunca fizeram ou não fazem uso regular de qualquer tipo de fumo. Dos 52 tabagistas ativos, 15,4\% evoluíram com algum tipo de complicação respiratória. Sendo que apenas $9,3 \%$ dos pacientes que nunca fumaram evoluíram com tal desfecho.

\begin{tabular}{|c|c|c|c|c|}
\hline \multirow[b]{2}{*}{ Variável } & \multirow[b]{2}{*}{ Categoria } & \multicolumn{3}{|c|}{ Complicação } \\
\hline & & $\begin{array}{c}\text { Sim } \\
\text { N (\%) }\end{array}$ & $\begin{array}{c}\text { Não } \\
\mathbf{N}(\%) \\
\end{array}$ & $\begin{array}{r}\text { Total } \\
\mathbf{N}(\%) \\
\end{array}$ \\
\hline $\begin{array}{l}\text { Características } \\
\text { pacientes }\end{array}$ & & & & \\
\hline Tabagismo ativo & $\begin{array}{l}\text { Sim } \\
\text { Não }\end{array}$ & $\begin{array}{l}8(15,4) \\
26(9,3)\end{array}$ & $\begin{array}{c}44(84,6) \\
255(90,7)\end{array}$ & $\begin{array}{c}52(100,0) \\
281(100,0)\end{array}$ \\
\hline
\end{tabular}

Tabela 2: Amostra de acordo com o tabagismo ativo. 


\section{DISCUSSÃO}

De acordo com estudo feito por Moura e colaboradores, em 2012, baseado no Sistema de Informações Ambulatoriais do SUS (SAI/SUS), houve uma média de 0,06 consulta/homem/ano em 2010, na faixa etária entre 20 e 59 anos de idade. Agravos relacionados à morbimortalidade masculina podem justificar maior frequência de atendimento desse público em nível secundário, frequência de $57 \%$ da amostra analisada, inferindo maior vulnerabilidade à ocorrência de complicações pós-operatórias, devido ao maior número de comorbidades clínicas associadas.

Ainda em termos comparativos, homens de 25 a 59 anos preferem utilizar serviços que respondam objetivamente às suas necessidades, como Pronto Atendimentos, em detrimento ao uso regular da atenção básica. Dessa maneira, foi instituída a Política Nacional de Atenção Integral à Saúde do Homem (PNAISH), como política pública, com o objetivo de melhoria das condições de saúde da população masculina do Brasil, responsabilizando-o pela sua própria saúde. Resultados da amostra analisada neste estudo corroboram os dados da literatura ao constatar que a maior frequência, $39,6 \%$, foi composta por adultos jovens, sendo que $13,2 \%$ dos homens evoluíram com algum tipo de complicação respiratória e apenas $6,3 \%$ das mulheres evoluíram com tal desfecho, proporcionando uma razão masculino/feminino de aproximadamente 2:1 .

Existem fatores sociais que fazem com que os homens não procurem pelo serviço de saúde com a mesma frequência que mulheres. Tem-se, muitas vezes, a mentalidade de que o homem deve ser forte, de forma que ficar doente seria sinônimo de fraqueza. Este tipo de ideologia acaba por prejudicar a ida de homens aos serviços de saúde, de forma que, quando apresentam alguma enfermidade, ou não possuem ciência da mesma, ou acabam por descobri-las de forma tardia.

Em revisões sistemáticas, os estudos vigentes mostram que o risco de complicações pulmonares aumenta progressivamente, conforme a faixa etária, com ponto de corte a partir dos 50 anos (MARTINS; VALADARES; TAVARES, 2016). Em consonância com esse estudo, foi possível observar que houve uma maior 
proporção de pacientes que evoluíram com complicação a partir de 60 anos de idade, com uma frequência de $20 \%$ do público dessa estrutura etária, ao mesmo tempo em que, nos adultos jovens de até 39 anos, apenas 5,3\%, e nos adultos com idade entre 40 e 59 anos, 6,9\% evoluíram com algum tipo de complicação respiratória. Isso pode dever-se ao fato de que a elevada faixa etária pode associarse com comorbidades crônico-degenerativas, aumentando, dessa forma, o risco de evoluir com desfechos negativos no pós-operatório.

Apesar de um Programa Nacional de controle do Tabagismo (PNCT), criado em 1989, é observado o desafio das ações de controle do tabagismo se firmarem e a manutenção de elevado índice de tabagistas ativos, sendo um grave problema de saúde pública e predispondo à ocorrência de patologias associadas, principalmente no que tange às Doenças Crônicas Não Transmissíveis (DCNT). De acordo com Martins, Valadares e Tavares, em 2016, história de tabagismo com mais de 20 maços/ano prediz alto risco de complicações pulmonares, o que também condiz com os resultados encontrados na população analisada, visto que a ocorrência de complicações em pacientes tabagistas ativos foi de 15\%, em comparação aos não tabagistas, apenas $9 \%$, o que corresponde a 1,65 vezes mais ocorrências em fumantes.

É importante frisar que $15 \%$ da amostra autodeclararam tabagistas ativos, o que corresponde a uma proporção aproximada da média brasileira, de acordo com um estudo publicado por Facina, em 2011, com base em dados do livro Pesquisa Especial de Tabagismo (PETab) - Relatório Brasil, lançado em parceria entre o Instituto Nacional do Câncer (INCA) e Organização Pan-americana de Saúde (OPAS), no qual mostrou que $17,2 \%$ da população adulta brasileira era tabagista ativa, com uma prevalência de $21,6 \%$ entre os homens e $13,1 \%$ entre as mulheres. Dessa forma, é possível relacionar tais dados com a maior frequência de complicações respiratórias no pós-operatório na população masculina também em decorrência da maior proporção de tabagistas ativos. 


\section{CONCLUSÃO}

Ao analisar o perfil sociodemográfico, mostrou-se que a maioria dos pacientes era do sexo masculino e com faixa etária predominante de adultos jovens, com idade até 39 anos, sendo uma parcela significativa de não tabagistas ativos. Apenas 10\% da amostra evoluíram com alguma complicação respiratória no pós-operatório. Sendo válido ressaltar, que desses, houve uma maior proporção de tabagistas ativos ao compará-los com os que nunca fumaram. A partir dos resultados obtidos, é possível inferir que as características encontradas na população estudada podem contribuir para a organização da assistência, uma vez que permitem identificar os grupos populacionais mais vulneráveis.

Destaca-se que o fato de haver menos mulheres que apresentam complicações pode estar relacionado ao menor número de mulheres tabagistas e à maior procura feminina pelos serviços de atenção básica, o que ajuda a prevenir e tratar doenças crônicas, que são fatores de risco para o desfecho clínico de complicação respiratória pós-operatória.

Por se tratar de um estudo retrospectivo, o presente estudo apresenta algumas limitações, como: uso de poucas variáveis sócio-demográficas; análise de prontuários de apenas um hospital da região; e não ter utilizado outras variáveis, como histórico de doenças, complicações apresentadas, avaliação clínica do paciente, dentre outras.

Sugerem-se estudos mais complexos e com mais variáveis a serem analisadas, para ampliar a compreensão da realidade e perfil dos usuários do SUS em nível regional. 


\section{REFERÊNCIAS BIBLIOGRÁFICAS}

ÁVILA, Ana Carolina de; FENILI, Romero. Incidência e fatores de complicações pulmonares pós-operatórias em pacientes submetidos à cirurgias de tórax e abdome. Revista Colégio Brasileiro Cirurgia. v. 44, n. 3, p. 284-292, 2017. Disponível em: $<$ http://www.scielo.br/scielo.php?script=sci_abstract\&pid=S0100-

69912017000300284\&lng=pt\&nrm=iso>. doi: https://dx.doi.org/10.1590/0100-69912017003011.

BRASIL. Conselho Nacional de Saúde. Resolução N 466, de 12 de Dezembro de 2012. Disponível

<https://bvsms.saude.gov.br/bvs/saudelegis/cns/2013/res0466_12_12_2012.html>.

em:

BRASIL. Instituto Nacional de Câncer. Organização Pan-Americana da Saúde. Pesquisa Especial de Tabagismo - PETab: relatório Brasil. Rio de Janeiro: INCA, 2011. 205 p.

LANGERON, Olivier et al. Postoperative pulmonary complications updating. Ann Fr Anesth Reanim. v. 33, n. 7-8, p. 480-483, 2014. Disponível em: <https://www.ncbi.nlm.nih.gov/pubmed/25168300>. https://doi.org/10.1016/j.annfar.2014.07.741.

MARTINS, Amanda Cristina de Oliveira; VALADARES, Roberto José; TAVARES, Anamaria Ruiz Combat. Avaliação pré-operatória no paciente pneumopata. Rev Med Minas Gerais. v. 26, supl 7, p. S45-S52, 2016.

MOURA, Erly Catarina et al. Perfil da situação de saúde do homem no Brasil. 1. ed. Rio de Janeiro: Fundação Oswaldo Cruz - Instituto Fernandes Figueira, 2012. 130 p.

SILVA, Denise Rossato; KNORST, Marli Maria; GAZZANA, Marcelo Basso. Valor dos achados clínicos e da avaliação funcional pulmonar pré-operatórios como preditores das complicações pulmonares pós-operatórias. Rev Assoc Med Bras. v. 56, n. 5, p. 551-557, 2010. Disponível em: $\quad<h t t p: / / w w w . s c i e l o . b r / s c i e l o . p h p ? s c r i p t=s c i=a b s t r a c t \& p i d=S 0104-$ 42302010000500016\&lng=pt\&nrm=iso\&tlng=pt>. doi: https://dx.doi.org/10.1590/S010442302010000500016 .

SILVA, Zilda Pereira da et al. Perfil sociodemográfico e padrão de utilização dos serviços de saúde do Sistema Único de Saúde (SUS), 2003-2008. Ciênc saúde coletiva. v. 16, n. 9, p. 3807-3816, 2011. Disponível em: <http://www.scielo.br/scielo.php?pid=S141381232011001000016\&script=sci_abstract\&tlng=pt>. doi: https://dx.doi.org/10.1590/S141381232011001000016.

TAYLOR, Amber; DEBOARD, Zachary; GAUVIN, Jeffrey Mark. Prevention of postoperative pulmonary complications. Surg Clin North Am. v. 95, n. 2, p. 237-254, 2015. Disponível em: <https://www.ncbi.nlm.nih.gov/pubmed/25814104>.

https://doi.org/10.1016/j.suc.2014.11.002.

YANG, Chun Kevin et al. Pulmonary complications after major abdominal surgery: National Surgical Quality Improvement Program analysis. J Surg Res. v. 198, n. 2, p. 441-449, 2015. Disponivel em: <https://www.ncbi.nlm.nih.gov/pubmed/25930169>. doi: https://doi.org/10.1016/j.jss.2015.03.028. 